\title{
DESIGN KNOWLEDGE REUSE IN DESIGN OF PROGRESSIVE STAMPING TOOLS: A QUALITATIVE STUDY
}

\author{
Jonsson, Carl-Johan; \\ Stolt, Roland; \\ Elgh, Fredrik \\ Jönköping University
}

\begin{abstract}
Progressive stamping tools are widely used in mass-production of sheet metal components and their performance is critical as the design of the tool impact the cost of the manufactured component significantly.

Knowledge reuse is an important part of successful design in general, and in progressive stamping tool design in particular. In the study described in this paper, 8 tool designers from 5 different Swedish companies were interviewed about (1) at what points in the tool design process they search for previously designed tools for information and knowledge reuse, (2) under what conditions and for what reasons does this reuse take place, and (3) what types of information and knowledge are sought for at each point. The results show that reuse of information and knowledge from previously designed tools happens in many parts of the tool design process. The reasons and conditions for reuse vary depending on where in the process the designer is. High component complexity is one example of a common factor triggering reuse. Also, information about the performance of the tool is important to tool designers, as they only want to reuse information and knowledge from tools with good performance and low maintenance.
\end{abstract}

Keywords: Design practice, Knowledge management, Design process, Design reuse, Stamping tools

\section{Contact:}

Jonsson, Carl-Johan

Jönköping University

Product development, Production and Design

Sweden

carl-johan.jonsson@ju.se 


\section{INTRODUCTION}

Progressive sheet metal stamping is a widely used method for manufacturing sheet metal parts in an economic, efficient, reliable, and industrialized manner, with a minimum number of manual post-processing steps. It is used in many different applications, such as computers, cars, luminaries, etc. The main principle is to conduct a series of basic sheet metal forming operations, such as bending and punching, in a step-by-step manner.

As many products have short lifecycles, and individualization and customization of products increases the product variance, there is increased pressure on manufacturing methods that have higher initial investment in terms of tools, such as progressive sheet metal stamping, to lower that investment along with the lead time for creating the equipment.

Traditionally, the process of designing and manufacturing sheet metal stamping tools has been characterized by being based on craftsmanship and a few key individuals with much experience. According to Leacock (2012), tool design for sheet metal forming is one of the areas where the knowledge and intuition of the expert still is the main source of know-how. Leacock (2012) also points to the increasing need to embed this knowledge and intuition within the tool development process. There exists many design-supports for aiding and automating tool design tasks, such as nesting, pilot placement, and analyzing and simulating various parts of the process such as formability analysis, final shape analysis, etc. Despite the existing supports for design and analysis, the tool designers frequently refer to previously designed tools looking for similar design solutions. The low usage of dedicated product data management systems (PDM) for the tools, makes the process of finding information about previous tools, to a large degree, dependent on the particular tool designer's ability to recall and find the information and documentation required (Jonsson et al., 2020). Therefore, this industry could benefit from having supports to enable tool designers to reuse knowledge and information in a more systematic manner.

Reuse of knowledge can be from different sources, such as memory, reports, colleagues, etc. For the sake of clarity, in this paper the term used for the type of reuse of information and knowledge from previously created tools, mainly via CAD-files, is "design knowledge reuse".

This paper presents the results of an interview study conducted with 8 tool designers at 5 Swedish companies. The objective of the study was to investigate the design knowledge reuse in tool design for sheet metal stamping, to get a better understanding of the details of it. This was done as part of a research project aimed at enhancing this reuse and making it more available, systematic, and structured. To this end, and to add to previous research in information and knowledge reuse, the objective was broken down into the following questions to capture the needed information:

- Where in the process does the design knowledge reuse take place?

- What triggers the designer to look for previous designs for input?

- What information and knowledge are sought for and in what formats?

Triggers, in this context, can be direct reasons for reuse, but also what conditions that causes reuse.

To answer the questions, the first in particular, the process of progressive stamping tool design will have to be described in more detail. This is done in Section 3.2.

\subsection{Terminology of stamping tools}

In Figure 1, a progressive stamping tool is shown and some of the parts that are referenced in this paper are named for increased clarity. The tools, sometimes referred to as "die sets", are mounted in large presses that move the upper half of the tool up and down. Each stroke of the press is creating (at least) one finished part. Between each stroke, the sheet metal strip is moved forward to the next station. To punch holes and cut contours, punches and dies are used. To hold the strip in place while forming and cutting operations take place, a spring-loaded stripper plate is used. The stripper plate also helps when pulling the punches back out again. To position the strip correctly before the stripper plate holds it in place, steering pins, called pilots, are pushed down into specifically punched pilot holes. Similarly, to punches, there are forming dies that have an upper and lower half. Because of forming operations, the strip is not flat all the way. To be able to feed the strip forward without interference, the strip needs to be lifted. This is often done by spring-loading parts in the lower die-set to push the strip up when the tool is open. To keep the upper and lower part of the tool correctly positioned relative to each other, and to take up some of the lateral forces from forming, guideposts are used. To verify that the finished components exit the tool correctly, ejection sensors or controllers are used. 


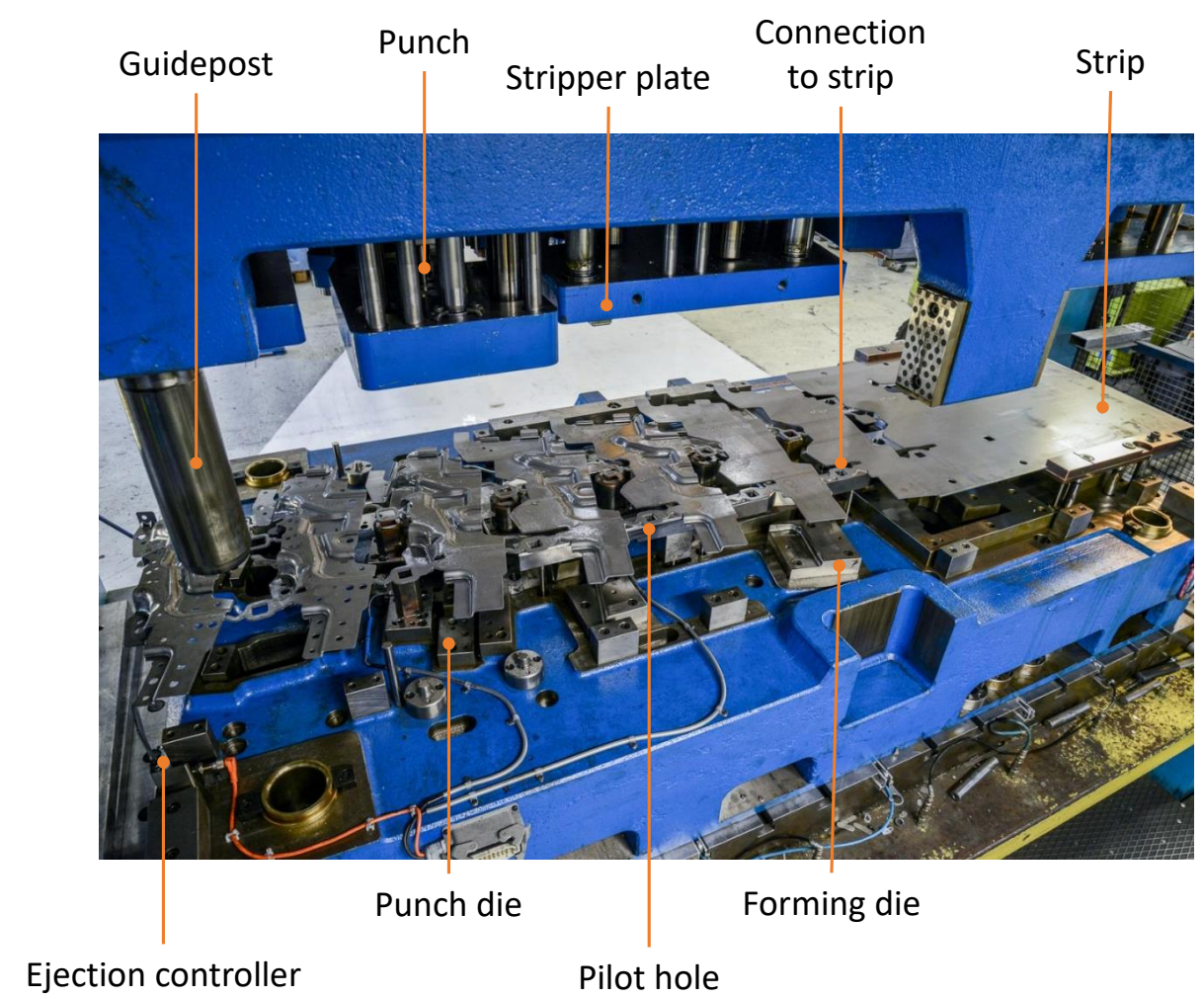

Figure 1. Example, progressive stamping tool for sheet metal.

\section{RELATED RESEARCH}

In this section a summary of previous research regarding information and knowledge use and reuse is presented. The information and knowledge reuse of engineers have been studied extensively during the past decades. From a selective review of this work some key points emerge that are described below. There is a consensus that engineers depend on internal sources to gain information rather than external to the organization. Such as colleagues, internal reports, existing drawings, etc. According to Allard et al. (2009) the size of the company does not significantly influence this. The engineers rely on people with experience and hands-on knowledge for gaining information, forming so-called social knowledge networks (Allard et al., 2009; Court, 1997; Demian and Fruchter, 2006).

The engineers' own experience and knowledge is a commonly used source of information, and/or knowledge about location of information (approximately 30\% of the usage) (Court, 1997). While the concept of "cost", in terms of ease of access and low effort, tends to contribute to the choice of source for information (Allard et al., 2009), the engineers are reluctant to organize and prepare information for future reuse. "Even where engineers do trust the systems, they are very often not willing to spend significant time organizing documents to assist their future access, owing to the difficulty of decision-making in this task" (McMahon et al., 2004).

Khadilkar and Stauffer (1996) observed that almost $50 \%$ of queries to a database tool for design information from previous activities during conceptual design was on a conceptual level of detail. They also mention that a possible explanation for this is that the task assigned to the subjects had conceptual design as the scope. Similarly Baya et al. (1992) found that during shorter detailed redesign tasks the queries was more detailed in nature. They also conclude that the form of the information gained dictates the nature of the following line of queries by designers. Kuffner and Ullman (1991) used question asking protocols to study designers during a redesign task. The results show that $47 \%$ of the questions and conjectures made were about the construction of a design object and $31 \%$ about the purpose and operation. From this we can see that different types of information are needed at different stages of design.

The types of information that engineers seek at different stages are technical and contextual, according to Allard et al. (2009). "Technical" being related to, for example, documentation on technical solutions, 
and "contextual" referring to information on the context of the design process, such as design rationale. This is very similar to the findings about how engineers in construction and architecture explore documentation of previous projects by Demian and Fruchter (2006). There, the engineers were said to explore the documentation in two dimensions, the project context dimension, and the evolution history dimension.

There are different perspectives about why internal and local information sources (e.g. own memory, colleagues, etc.) are preferred and more efficient than external sources (e.g. repositories, suppliers, etc.). Allard et al. (2009) suggest that the most influential factors for information-source choice are cost (in terms of accessibility), quality, and trustworthiness. Another way of describing this is done by Demian and Fruchter (2006), who describes motives of the choice for internal sources over external in terms of the requirements for finding the information, understanding the context dimension, and understanding the evolution dimension. Using the speed of accessing the own memory, the understanding and trustworthiness that comes with personal experience, and the richness of face-to-face communication, the choice of internal information sources become natural.

\section{STUDY DESCRIPTION AND METHOD}

Five companies were included in this study. The participating companies (listed in Table 1 are located in Sweden. The tools they design and produce, are also mostly used in production located in Sweden. Two different categories of companies that designs stamping tools has been identified. Both categories are represented in the study to account for eventual differences in the process of working with tool design. The categories are (1) companies that acts as OEMs, that have their own tooling departments along with product development and production, and (2) tooling companies (TC) that acts as suppliers to the manufacturing companies and only deliver the tools. Differences in the tool design process between these two categories are mostly associated with the OEM's tooling departments being able to participate earlier in the development process of the product they design tools for (Jonsson et al., 2020). Among the companies, there is also a wide range of components represented that they create tools for, regarding thickness, material, complexity, etc. The participants in the study ranged from experienced tool designers to tool

Table 1. Informaiton about the companies.

\begin{tabular}{|c|l|l|l|l|l|}
\hline Company & Category & $\begin{array}{l}\text { No. } \\
\text { employees }\end{array}$ & Tools / year & $\begin{array}{l}\text { No. } \\
\text { participants }\end{array}$ & $\begin{array}{l}\text { Experience of } \\
\text { participants }\end{array}$ \\
\hline C1 & TC & $20-30$ & $20-25$ & 1 & $15 \mathrm{yr}$ \\
\hline C2 & TC & $20-30$ & $25-30$ & 2 & $25 \mathrm{yr}$ and 35yr \\
\hline C3 & TC & $20-30$ & $40-45$ & 1 & $8 \mathrm{yr}$ \\
\hline C4 & OEM & Around 300 & $15-20$ & 2 & $9 \mathrm{yr}$ and $11 \mathrm{yr}$ \\
\hline C5 & OEM & Around 300 & $50-55$ & 2 & $35 \mathrm{yr}$ and 32yr \\
\hline
\end{tabular}

designers with less experience with the span between 8-35 years of experience in the business. Here, it is worth noting that, the tool designers with between 8-15 years of experience were referred to as newer in the industry by their colleagues. The choice of having varying experience among the tool designers was made with the following rationale: those with more experience might be better equipped to express their thinking in words, while a less experienced designer would be more prone to go back to previously designed tools when facing design challenges. A total of eight designers took part in the study.

\subsection{Method for data collection}

The data collection was done by using semi-structured interviews with each tool designer. The interviews were structured as a walk-through of the design process of an example tool, selected together by the participant and researcher. The researcher then asked questions about reuse of design information and knowledge from previously designed tools. The researcher asked follow-up questions if certain aspects of tasks were omitted by the designer. The scope of the questions was not limited to the example tool, but also allowed the questioning to include tool design in general, allowing the designers to draw from their experience. Understanding from (Jonsson et al., 2020), that the major design decisions and process planning of the tools are made in the earlier phases of tool design, was used to decide to focus the interviews and tool walk-though more on those early phases. 
Initially two pilot interviews were conducted where the purpose was to improve the interview guide and get a better understanding for the process steps, preliminary categories, and structure for the data analysis. The pilots were conducted at company $\mathrm{C} 2$ and C5 and had the same procedure as the main study. The outcome was additions to the list of tasks (Table 2) in the concept design phase used for structuring the interview and an increased understanding of the expectations of the results. All interviews were audio recorded and then the audio files were tagged and categorized by the interviewer, in order to create a data set.

Other methods to collect data that were considered for this study, but not used, were observation or "shadowing" (Czarniawska, 2007) and "think-aloud protocols" (Boren and Ramey, 2000). These methods were used in some of the studies referenced in Section 2.The advantage of using observations or think-aloud protocols is that, if done correctly, the data collected is not interpreted and filtered by the participant and is therefore more reliable. The data from the interviews in this study is the tool designer's own verbal reconstruction of the actions taken and thought process they had, during tool design. If the research questions of the study were more quantitative in nature, e.g., the frequency of reuse of design information and knowledge from previously designed tools, observations or think-aloud protocols would be preferable. In this study, however, the research questions are more qualitative in nature and asks more along the lines of, "when", "why" and "what". That requires more reasoning and follow-up questions to answer, and that type of reasoning is dependent on interpretation and the context of the specific case.

\subsection{Framework for coding and analysis}

As a starting point, previous knowledge about the stamping tool development process, as well as tool design handbooks, such as Suchy (2006), were used to construct a list of tasks that the conceptual tool design process consists of. The process of designing a progressive stamping tool can be divided into two steps: conceptual design (tool layout and process plan) and detailed design. Along with the input from the pilot interviews, a framework for questioning and starting point for analysis was established. The list of tasks is shown in Table 2. For the sake of brevity each task is assigned a reference in the form of a letter which is used in the paper. The detailed design step is more component centric, and therefore,

Table 2. List of tasks in tool desing.

\begin{tabular}{|c|l|}
\hline Reference & Conceptual Tasks \\
\hline A & Review of drawing tolerances and other requirements \\
\hline B & Formability evaluation/simulation \\
\hline C & Orientation of the part on the strip \\
\hline D & Part connection to the strip \\
\hline E & Piloting \\
\hline F & Approximate shape and number of punches \\
\hline G & Approximate shape and number of forming operations \\
\hline H & Tool station order, including idle stations \\
\hline I & Use of special solutions/functions \\
\hline J & Functionality and verification for lift of the strip \\
\hline K & Conceptual design of die set, punch and die holder, etc. \\
\hline L & Scrap removal and part ejection \\
\hline M & Material selection and surface treatment of tool components \\
\hline & Detailed design Tasks \\
\hline N & Punches and dies \\
\hline O & Forming dies \\
\hline P & Dimensioning and placement of springs \\
\hline Q & Punch and die holders, stripper plate \\
\hline R & Sensors and controllers \\
\hline S & Die set, guideposts, stop blocks, press mounting adaptions \\
\hline T & Special solutions/functionality \\
\hline
\end{tabular}


the list of tasks is mostly based on tool part names but grouped based on functionality. It is important to note that during tool design, the tasks are not necessarily performed in the order in Table 2, as the design process is iterative in nature and the significance of different tasks can vary from tool to tool.

In task "I" (Use of special solutions/functions), the tool designers identify the need for special functionality in the tool, to be able to manufacture the specific features of the component. These solutions are often standardized (if developed in-house) or bought from suppliers. Some examples are threading units, cam units to punch holes laterally, solutions for moving dies when bending more than $90^{\circ}$, etc.

\section{RESULTS}

\subsection{Occurances of reuse}

The occurrence of design knowledge reuse in the process of designing a progressive stamping tool among the tool designers in this study is shown in Figure 2 (tasks A-M) and Figure 3 (tasks N-T). They show the situations where design knowledge reuse is performed regarding a particular task. All of the

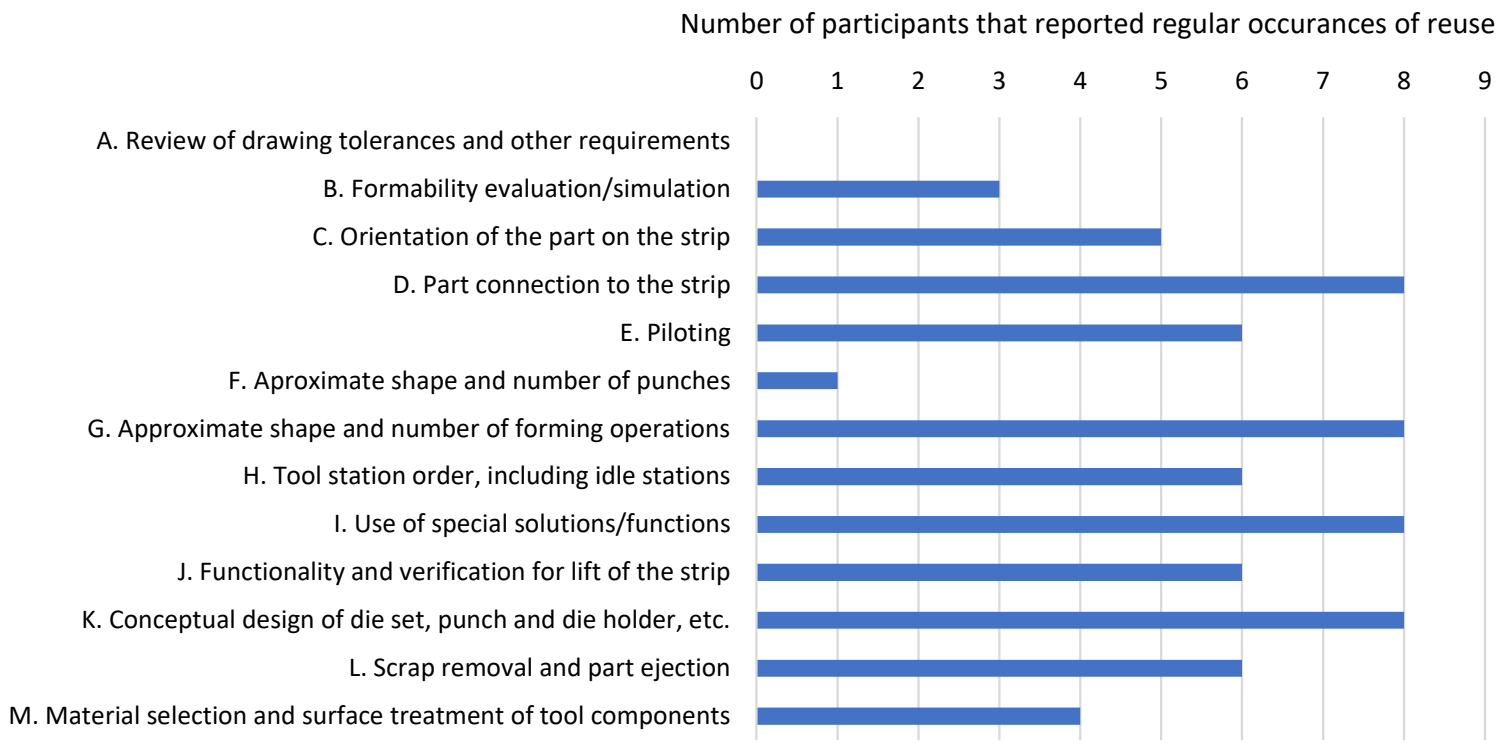

Figure 2. Occurance of reuse in each conceptual design task.

participants reported reuse in tasks "D", "G", " $\mathrm{I}$ ", "O" and "T". Regarding task "K", for the design of die set and the adaption of mounting the tool in a specific press, all companies used templates for all presses they design tools for. This was interpreted as design knowledge reuse in this context and is the main reason for the high value.

Some of the tasks were seen by the tool designers as more of a "puzzle to solve". There, the tool designer have to find a way forward by only being able to use parameters that are unique to the current part and problem. This was said about tasks "C", "E", "F", and "H". It should be noted that Figure 2 and Figure 3 does not give any information about the frequency of reuse in each task. All design knowledge reuse the participants do, is completely based on them knowing, what they need, already exists. If they do not know what to look for, or do not think they can find what they are looking for, in timely manner, they will not search for a tool to reuse from. With this background, the participants were asked to estimate their own frequency of design knowledge reuse. The answers ranged from: once every other tool design, to 4-5 times per tool design. It has to be stressed that those are estimates but they can give an idea of the order of magnitude of the reuse.

\subsection{Triggers for reuse}

The triggers mentioned for reuse in each task is shown in Table 3. It gives some insight to why reuse is conducted, along with what information was searched for. The tasks that no participants conducted any reuse in, are removed from the list of tasks in this table. A pattern that was observed regarding the 


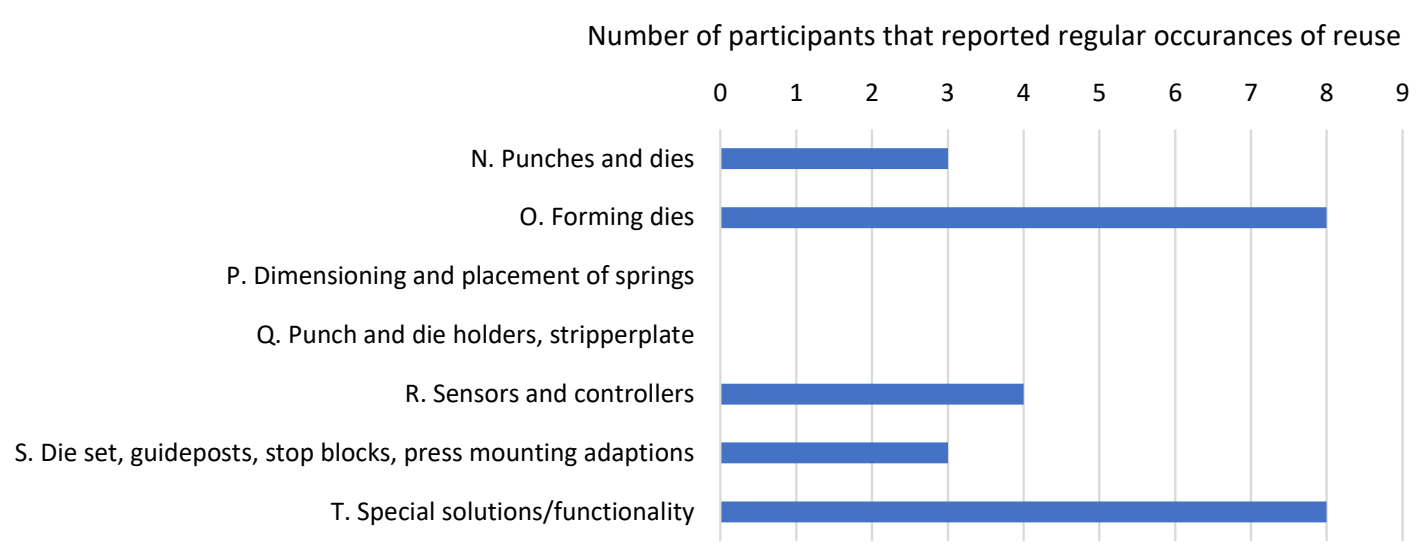

Figure 3. Occurance of reuse in each detailed design task.

triggers, was that some of the companies have an informal set of design solutions they applied in most cases, for some of the tasks. These tasks are, "D", "J", and for some variants of "I", such as overbending upwards. As an example of this, when first being asked about the connection of the part to the strip, the tool designers said: every part is unique, and the solution is completely dependent on the shape of the part. But after some follow-up questions, it became clear that 2-4 different principal solutions for connection existed, and the companies even had informal names for the different variants (although different for each company).

High part complexity and requirements through tolerances are generally challenging in tool design. Part complexity and tolerances are features that are unique, to some degree, from part to part. It is not obvious that they should be triggers of design knowledge reuse. In this context, part complexity has two dimensions. The first is the number of features of the part, e.g., holes and bends. The second is the difficulty to manufacture any feature, where drawn features would be considered complex. In practice, however, it is sometimes possible for a tool designer to break the problem down into smaller parts, and then reuse knowledge and information from previous tools to solve the more generic sub-problems.

Another common trigger for reuse, is when proposed design alternatives are evaluated and it is not clear that the solution is feasible. In that case, the tool designer looks for precedence among similar examples of this solution being used in previously made tools. An example of this could be, if the geometry of the part is such that piloting holes would have to be punched in the connecting strip between parts, while being very close to the geometry of the finished part. This would trigger the tool designer to search for a previous tool where this have been done and confirm whether it is possible or will cause faulty parts. "Similar part" as a trigger is perhaps the most obvious, but it also has many facets to it. There are many ways two sheet metal parts can be similar. It is not within the scope of this paper to delve deeper into the details of this, except to recognize that there are different types of similarities. Example of this is: similar geometric features, similar in size, similar in a characteristic section or profile, scale versions of one another, similar topology, etc.

\subsection{Types of information}

The categories of information the tool designers looked to reuse, that were identified, are mainly: solution ideas, company standard solutions, dimensions, and CAD-geometry. "Solution ideas" is the type that is involved in the most tasks. When reusing a solution idea, it is the principles of a specific design that are reused. The tool designer must analyze the tool for reuse, extract the general idea for the solution, and adapt it to the new conditions. It involves similarity in the problem situation, rather than similarities of the parts being manufactured. Reusing by company standard solutions is very similar to reusing solution ideas. The main difference is that the extraction of the general idea for each solution has already been made. The tool designer only needs to select and adapt the solution to the current tool. The reuse of specific dimensions from previously made tools can be exemplified by checking what clearances and radii were used in a die to create a collar with a certain shape, or what compensation were used for spring back. CAD-geometry can be reused in different types of situations. Either there are templates that the company has prepared to speed up repetitive modelling tasks and to get uniform structure of all 
Table 3. Triggers for reuse and sought for information types.

\begin{tabular}{|c|c|c|}
\hline Task & Triggers & Information type \\
\hline $\mathrm{B}$ & Double check simulation, Similar shape of part & Verification \\
\hline $\mathrm{C}$ & $\begin{array}{l}\text { No obvious preferred orientation, Similar shape of } \\
\text { part }\end{array}$ & Solution idea \\
\hline $\mathrm{D}$ & $\begin{array}{l}\text { Special requirements from customer, Existing prin- } \\
\text { cipal solution, No obvious solution, Similar shape } \\
\text { of part }\end{array}$ & Solution idea \\
\hline $\mathrm{E}$ & $\begin{array}{l}\text { No obvious solution, Tolerance requirements, Not } \\
\text { enough space }\end{array}$ & Solution idea \\
\hline $\mathrm{F}$ & Customer requirements & Solution idea \\
\hline G & $\begin{array}{l}\text { Narrow tolerances, Need for multiple operations, } \\
\text { Overbending, Spring back, Similar shape of part }\end{array}$ & $\begin{array}{l}\text { Solution idea, CAD-geometry, } \\
\text { Dimension for spring back com- } \\
\text { pensation, Number of steps, Total } \\
\text { function solution }\end{array}$ \\
\hline $\mathrm{H}$ & $\begin{array}{l}\text { Feasibility of multiple operations in a single step, } \\
\text { Tight space between punches, High part complexity }\end{array}$ & $\begin{array}{l}\text { Solution idea, Possible minimum } \\
\text { dimensions }\end{array}$ \\
\hline $\mathrm{I}$ & Need for special solutions & $\begin{array}{l}\text { Solution alternatives, Space } \\
\text { requirements }\end{array}$ \\
\hline $\mathrm{J}$ & Standard solutions not applicable & Solution idea, CAD-geometry \\
\hline $\mathrm{K}$ & Strive for standardized solutions, Cast die sets & $\begin{array}{l}\text { Design principals, CAD-geometry, } \\
\text { Dimensions }\end{array}$ \\
\hline $\mathrm{L}$ & Flat parts, Small parts, "Almost every tool" & Solution idea \\
\hline $\mathrm{M}$ & Tough forming operations, Customer requirements & $\begin{array}{l}\text { Material, Hardening, Surface treat- } \\
\text { ment }\end{array}$ \\
\hline $\mathrm{N}$ & Complex punch geometry, material and thickness & Clearance, Dimensions \\
\hline $\mathrm{O}$ & $\begin{array}{l}\text { Complex shapes, Spring back, progressive draw, } \\
\text { Space restrictions }\end{array}$ & $\begin{array}{l}\text { Solution idea, CAD-geometry, } \\
\text { Dimensions }\end{array}$ \\
\hline $\mathrm{R}$ & Standard solutions not applicable & Solution idea \\
\hline $\mathrm{S}$ & Strive for standardized solutions, Cast die sets & $\begin{array}{l}\text { Design principals, CAD-geometry, } \\
\text { Dimensions }\end{array}$ \\
\hline $\mathrm{T}$ & Need for special solutions & $\begin{array}{l}\text { Features and dimensions for solu- } \\
\text { tion and mounting, Reseller }\end{array}$ \\
\hline
\end{tabular}

designed tools, or there are parts of the CAD-files from a previous tool that can be copied and reused with only minor adaption.

It can be noted that solution ideas are more prevalent in the conceptual phase, and less so in detailed design. Likewise, the opposite can be observed for dimensions.

\section{DIsCUSSION}

The design knowledge reuse, in this study, is most prevalent in two different types of tasks: tasks that have standardized solutions (" $D$ " and " $K$ "), and tasks that often prove challenging and have aspects that are unique to each new tool ("G", "I", "O", and "T"). It seems that it is easier for the tool designers to reuse design knowledge in cases where they can clearly formulate what they are looking for. In the case of "C", it can be difficult to articulate what constitutes a similar shape and find useful examples from past tools. In contrast, in "I", there is often clear parameters (e.g., the usage of a threading station) that matches the new case with an old tool.

The concept of "cost" (Allard et al., 2009), and how it affects the reuse is clear, since if tool designers do not know beforehand where to find a previous tool, they will likely try to solve the task at hand without help from reuse. For the tool designers in this study, finding the tool they are looking for can be difficult and time consuming. The companies store their CAD-files for the stamping tools on a network drive with folders structures for customer or product. Observing how the search for a specific tool was carried out, the use of visual representations of the tool with pictures was found to quickly allow the 
designers to evaluate if it was the correct tool. However, if the designer is not aware that a tool that could be interesting exists, reuse is not going to happen. The reuse can also be compared between the tool designers with less experience and those with more. While the amount of data is small, the tendency is that the tool designers with more experience reused more often. An explanation for this could be, that they have seen more tools and know better what already exists. All participants, however, commented that they would reuse more if they could find tools based on the information they had at hand, which varies from project to project.

The triggers for design knowledge reuse, mostly revolve around when the tool designer does not intuitively know what solution is best for the current situation. This occurs either when the proposed solution is close to a limit for what is feasible, when the complexity is too high, or when two or more options seem equally good. The other situations that trigger reuse are when companies have standard solutions to certain problems, or templates for certain geometries. In the non-standard cases, the tool designer would also have to estimate the transferability of the knowledge gained from reuse, in order to determine if it should be pursued. This ties into the concepts of "cost" (in terms of accessibility), quality, and trustworthiness of information (Allard et al., 2009). However, the quality aspect is less obvious since all reused information comes from CAD-files and are of similar quality. The role of trustworthiness is one of the most significant in this study, and all participants commented on this during the interviews. They would only want to reuse design knowledge from tools that have been proven to work well in production, or required few iterations of testing and revision. Unless they already knew if the tool had been working well, they would check with other tool designers, production engineers, maintenance personnel or the maintenance system before reusing solutions. This is similar to understanding the evolution history of projects Demian and Fruchter (2006).

The tool designers mostly sought to reuse solution ideas and conceptual solution alternatives to the problems they encounter. This study confirms the results of previous research by Baya et al. (1992) and Khadilkar and Stauffer (1996), in that solution ideas, that are more conceptual in nature, are more prevalent in the conceptual phase of design. Similarly, dimensions and information about mounting hole patterns, etc., is more prevalent in the detailed design phase.

\section{LIMITATIONS}

When evaluating the results of the study, the following limitations must be considered.

Much of the data collected is the participants remembering and retelling their view of past events. Other parts of the data are more of the type where the participants tell their reasoning for different example situations. There is always a discrepancy between reality and what a participant remembers and can articulate.

Differences in how much design knowledge reuse there is at a given company or conducted by an individual designer can depend on several different factors. These factors can be, large variations in part shape and geometry from project to project, variations in part thickness from project to project, the number of tools designed per year, complexity level of parts, experience of designer. Similarly, the same can be said for variations in what tasks reuse is most prevalent.

\section{CONCLUSIONS AND FUTURE RESEARCH}

Design knowledge reuse in the design of progressive stamping tools is conducted in many parts of the process. In order to enhance this reuse, designers would have to be support in different ways in different situations. The triggers for reuse and the type of information wanted, vary depending on the conditions of the current situation. This study gives insights into how these needs vary in different parts of tool design.

The trustworthiness and the information about performance of solutions in the reuse is of great importance to the tool designers. This is used as a mechanism for not reusing mistakes and only reuse best practices in an implicit way.

Future research will be focused on developing supports for enhancing this type of reuse. That area has many challenges that have been studied previously, but there is still room for improvement in the areas of methods to efficiently record and structure design information to enable search, and methods and interfaces to facilitate finding and reusing based on the varying conditions of the situation. 


\section{ACKNOWLEDGMENTS}

This study was part of a research project funded by Stiftelsen Seydlitz MP bolagen. The authors would also like to thank the participating companies.

\section{REFERENCES}

Allard, S., Levine, K.J. and Tenopir, C. (2009), "Design engineers and technical professionals at work: Observing information usage in the workplace", Journal of the American Society for Information Science and Technology, Vol. 60 No. 3, pp. 443-454.

Baya, V., Gevins, J., Baudin, C., Mabogunje, A., Toye, G. and Leifer, L. (1992), "Experimental study of design information reuse", in: 4th International Conference on Design Theory and Methodology, Scottsdale, AZ, USA, 13-16 September 1992, American Society of Mechanical Engineers, Design Engineering Division (Publication) DE, pp. 141-147.

Boren, T. and Ramey, J. (2000), "Thinking aloud: Reconciling theory and practice", IEEE transactions on professional communication, Vol. 43 No. 3, pp. 261-278.

Court, A. (1997), "The relationship between information and personal knowledge in new product development", International journal of information management, Vol. 17 No. 2, pp. 123-138.

Czarniawska, B. (2007), Shadowing: and other techniques for doing fieldwork in modern societies, Copenhagen Business School Press DK.

Demian, P. and Fruchter, R. (2006), "An ethnographic study of design knowledge reuse in the architecture, engineering, and construction industry", Research in Engineering Design, Vol. 16 No. 4, pp. 184-195.

Jonsson, C.J., Stolt, R. and Elgh, F. (2020), "Stamping tools for sheet metal forming: Current state and future research directions", in: 27th ISTE International Conference on Transdisciplinary Engineering, Warsaw, Poland, 1-10 July 2020, IOS Press, pp. 281-290.

Khadilkar, D.V. and Stauffer, L.A. (1996), "An experimental evaluation of design information reuse during conceptual design", Journal of Engeering Design, Vol. 7 No. 4, pp. 331-339.

Kuffner, T.A. and Ullman, D.G. (1991), "The information requests of mechanical design engineers", Design studies, Vol. 12 No. 1, pp. 42-50.

Leacock, A.G. (2012), "The future of sheet metal forming research", Materials and Manufacturing Processes, Vol. 27 No. 4, pp. 366-369.

McMahon, C., Lowe, A. and Culley, S. (2004), "Knowledge management in engineering design: personalization and codification", Journal of Engineering Design, Vol. 15 No. 4, pp. 307-325.

Suchy, I. (2006), Handbook of die design, Vol. 1998, McGraw-Hill New York. 